\title{
Relação entre o Tratamento com Metformina e o Desenvolvimento de Hiperlactacidemia no Serviço de Urgência
}

\author{
Relation between Metformin Treatment and the Development of \\ Hyperlactacidemia at the Emergency Room
}

\author{
Daniela GUELHO ${ }^{1}$, Isabel PAIVA ${ }^{1}$, Francisco CARRILHO ${ }^{1}$ \\ Acta Med Port 2014 Mar-Apr;27(2):196-203
}

\section{RESUMO}

Introdução: Em diabéticos tipo 2 sob metformina o desenvolvimento de hiperlactacidemia ou mesmo acidose láctica parece decorrer de um evento precipitante agudo. Este estudo objetiva avaliar a prevalência e risco relativo de hiperlactacidemia em diabéticos observados no Serviço de Urgência, os fatores preditores da concentração de lactatos e a influência da lactacidémia no prognóstico. Material e Métodos: Estudo observacional transversal incluindo 138 diabéticos tipo 2, dos quais 66 sob metformina, e 83 doentes não diabéticos atendidos entre junho e outubro de 2012. Variáveis estudadas: idade, sexo, motivo de urgência, pressão arterial, hábitos farmacológicos, antecedentes, estudo analítico (bioquímica e gasometria arterial com doseamento de lactatos) e destino após observação. Análise estatística realizada com SPSS $21.0^{\circledR}$.

Resultados: A concentração de lactatos e proporção de hiperlactacidemia foram significativamente superiores nos diabéticos $(2,1 \pm$ $0,1 \mathrm{mmol} / \mathrm{L}$ vs $1,1 \pm 0,1 \mathrm{mmol} / \mathrm{L}, p<0,001$ e $39,1 \%$ vs $3,6 \%, p<0,001$, respetivamente); particularmente naqueles sob metformina comparativamente aos restantes diabéticos $(2,7 \pm 0,2 \mathrm{mmol} / \mathrm{L}$ vs 1,6 $\pm 0,1 \mathrm{mmol} / \mathrm{L}, p<0,001$ e $56,9 \%$ vs $23,3 \%, p<0,001$, respetivamente). Os diabéticos sob metformina contabilizaram uma probabilidade de hiperlactacidemia $25 x$ superior $(O R=25,10, p<0,05)$. A creatinina representou o único fator preditor independente da lactacidemia $(B=1,33 ; p<0,05)$. Os doentes com hiperlactacidemia apresentaram probabilidade $4,4 x$ superior de serem internados ou falecerem $(\mathrm{OR}=4,37, p<0,05)$. E, quando internados, contabilizaram tempo de internamento $(21,66 \pm 5,86$ dias vs $13,68 \pm 5,33$ dias, $p<0,001)$ e número de óbitos superiores $(12,5 \%$ ( $n=4)$ vs $4,3 \%(n=2), p<0,05)$.

Conclusões: Demonstrou-se um risco acrescido de hiperlactacidemia em diabéticos tipo 2, particularmente naqueles sob metformina. A creatinina sérica representou o único fator preditor independente da concentração de lactatos. A presença de hiperlactacidemia condicionou um pior prognóstico.

Palavras-chave: Diabetes Mellitus Tipo 2; Metformina; Hiperlactacidemia; Portugal; Serviço de Urgência.

\section{ABSTRACT}

Introduction: In type 2 diabetic patients treated with metformin the development of hyperlactacidemia or even lactic acidosis seems to result from an acute precipitating event. This study aims to assess the prevalence and relative risk of hyperlactacidemia in diabetic patients admitted in the Emergency Room, the predictive factors for high lactate concentration and the influence of hyperlactacidemia in patients' prognosis.

Material and Methods: Transversal observational study including patients observed between June and October $2012: 138$ type 2 diabetics, 66 treated with metformin, and 83 non-diabetic patients. Studies' variables: age, sex, cause of admition, blood pressure, drugs, personal history, analytical study (biochemistry and arterial blood gas analyses with lactate) and destination. Statistical analysis was performed using SPSS $21.0^{\circledR}$.

Results: Mean lactate concentration and hyperlactacidemia prevalence were significantly higher in diabetic patients $(2.1 \pm 0.1 \mathrm{mmol} / \mathrm{L}$ vs $1.1 \pm 0.1 \mathrm{mmol} / \mathrm{L}, p<0.001$ and $39.1 \%$ vs $3.6 \%, p<0.001$, respectively) and in those under metformin compared to other diabetics $(2.7 \pm 0.2 \mathrm{mmol} / \mathrm{L}$ vs $1.6 \pm 0.1 \mathrm{mmol} / \mathrm{L}, p<0.001$ and $56.9 \%$ vs $23.3 \%, p<0.001$, respectively). Diabetics on metformin presented a 25 -fold increased risk of hyperlactacidemia $(O R=25.10, p<0.05)$. Creatinine was the only independent predictive factor for lactate concentrations $(B=1.33, p<0.05)$. Patients with hyperlactacidemia had 4.4 times higher odds of being hospitalized or dying $(\mathrm{OR}=$ $4.37, p<0.05)$. When hospitalized, they had longer hospitalization periods $(21.66 \pm 5.86$ days vs $13.68 \pm 5.33$ days, $p<0.001)$ and higher rate of deaths $(12.5 \%(n=4)$ vs $4.3 \%(n=2), p<0.05)$.

Conclusion: There was an increased risk of hyperlactacidemia in patients with type 2 diabetes, particularly for those under metformin. Serum creatinine represented the only independent associated factor of lactate concentration. The presence of hyperlactacidemia was associated with worse prognosis.

Keywords: Metformin; Diabetes Mellitus, Type 2; Lactates; Emergency Service, Hospital; Portugal.

\section{INTRODUÇÃO}

A metformina reúne consenso entre as principais recomendações internacionais como terapêutica inicial da diabetes mellitus tipo 2 (DMT2) $\cdot^{1-4} \mathrm{~A}$ capacidade de diminuir a glicemia sem condicionar hipoglicemia, associar múltiplos benefícios metabólicos e simultaneamente apresentar um bom perfil de segurança justificam a eleição deste fármaco no tratamento da diabetes. O seu principal mecanismo antidiabético resulta da redução da produção hepática de glicose (neoglicogénese), desempenhando ainda funções como insulinossensibilizador, modulador de componentes do eixo das incretinas, redutor da absorção gastrointestinal de glicose e promotor indireto da função da célula $\beta$. A ação preferencial da metformina no hepatócito resulta do aumento da expressão de OCT1 (organic cation transpor-

1. Serviço de Endocrinologia, Diabetes e Metabolismo. Centro Hospitalar e Universitário de Coimbra. Coimbra. Portugal.

Recebido: 20 de Maio de 2013 - Aceite: 09 de Dezembro de 2013 | Copyright @ Ordem dos Médicos 2014 
ter 1) nestas células. A nível intra-celular, o fármaco parece exercer efeito através da inibição do complexo 1 da cadeia respiratória mitocondrial, que reduzindo o estado energético celular, ativa o sensor AMPK (AMP activated protein cinase) e afeta o programa neoglicogénico. ${ }^{5}$

O efeito adverso mais grave associado à utilização de metformina é a acumulação de ácido láctico e o desenvolvimento de acidose. Os níveis plasmáticos de lactato resultam de um balanço entre a sua síntese e utilização. O ácido láctico é produzido no músculo, tecido adiposo e fígado através do metabolismo anaeróbio da glicose. É rapidamente tamponado, em parte através do bicarbonato extracelular, resultando na formação de lactato. Sofre então metabolização hepática, ou, em menor extensão, renal, de forma a regenerar novamente piruvato, que por sua vez é convertido em dióxido de carbono e água ou em glicose, esta última resultante do processo da neoglicogénese. ${ }^{6}$

Ao condicionar uma inibição da neoglicogénese, uma predisposição ao metabolismo anaeróbio e a produção de lactato no intestino, a metformina poderá condicionar um aumento dos níveis séricos de lactatos nos doentes diabéticos. ${ }^{7}$ No entanto, parece ser um evento agudo que predispõe ao desenvolvimento hiperlactacidemia ou mesmo de acidose láctica. $\mathrm{O}$ mecanismo de produção de acidose láctica associado à metformina não se encontra completamente elucidado, mas parece ocorrer em contexto de intercorrências agudas que prejudiquem a capacidade de eliminação do lactato, de condições que aumentando os níveis plasmáticos de lactato, induzam a sua acumulação em contexto de uma clearance reduzida pela administração crónica de metformina (p.ex. sépsis, isquémia, etc.) ou ainda de situações que coincidem com uma elevação severa dos níveis séricos do fármaco. ${ }^{8}$

Fatores não relacionados diretamente com o tratamento da diabetes mellitus, como o peso corporal, a pressão arterial, a glicemia, a função renal e a utilização de anti-inflamatórios não esteróides ou de inibidores da enzima de conversão da angiotensina também parecem influenciar os níveis de lactatos. ${ }^{9-11}$ Existe, assim, alguma controvérsia na afirmação de que a utilização de metformina per si, condiciona um aumento dos níveis de lactato sérico nos doentes diabéticos.

Apesar dos recentes avanços terapêuticos e da evolução das técnicas de hemodiálise, a mortalidade associada à acidose láctica permanece muito elevada. ${ }^{12,13} \mathrm{E}$, mesmo que não condicione necessariamente o desenvolvimento de acidose, nos doentes em estado crítico, a hiperlactacidemia relativa associa-se a um aumento da mortalidade hospitalar. ${ }^{12}$ Como a maioria dos doentes, quer com hiperlactacidemia quer com acidose láctica, são assintomáticos, torna-se extremamente importante identificar marcadores fidedignos que evidenciem um risco acrescido para o seu desenvolvimento.

O objetivo do presente trabalho foi: avaliar a prevalência de hiperlactacidemia em doentes com DMT2 observados no Serviço de Urgência (SU); calcular o risco relativo de hiperlactacidemia nos doentes diabéticos sob metformina; identificar fatores preditores independentes da concentração de lactatos; e determinar a influência da lactacidémia no prognóstico destes doentes.

\section{MATERIAL E MÉTODOS \\ População de estudo e fontes de dados}

Estudo observacional transversal com inclusão aleatória de doentes com DMT2 que recorreram ao SU entre junho e outubro de 2012, por variados motivos não relacionados com a diabetes, que apresentassem bioquímica (com avaliação da função renal, hepática e doseamento de proteína $C$ reativa ultrassensível) e gasometria arterial (com doseamento de lactatos), realizadas na altura da admissão. Foram selecionados como controlos, doentes não diabéticos observados no SU durante o mesmo período de tempo. Foram considerados os doentes com o diagnóstico de diabetes tipo 2 se: em tratamento com dieta e/ou antidiabéticos orais há mais de seis meses, independentemente da idade; se mais de 60 anos na altura do diagnóstico independentemente do tipo de tratamento; doentes entre os 40 e os 60 anos sem introdução de insulina nos três meses após o diagnóstico de diabetes e com IMC superior a $30 \mathrm{~kg} / \mathrm{m}^{2}$.

Critérios de exclusão: diabéticos tipo 1 ou outras causas secundárias de diabetes; grávidas; condições previamente conhecidas que contraindicassem o tratamento com metformina particularmente insuficiência cardíaca classe III ou IV da classificação de New York Heart Association, insuficiência renal crónica (taxa de filtração glomerular basal $<60 \mathrm{~mL} / \mathrm{min} / 1,73 \mathrm{~m}^{2}$ ), insuficiência hepática (ou valores de transaminases hepáticas $>3 \mathrm{x}$ o limite superior do normal) ou insuficiência respiratória crónica (ou valores de paO2 $<25 \mathrm{mmHg}$ ); doentes transplantados renais ou hepáticos; eventos cardiovasculares agudos ou falência cardíaca; hipovolémia ou instabilidade hemodinâmica; alcoolismo ativo; infeção HIV; sépsis; trauma agudo ou procedimento cirúrgico major; síndrome do intestino curto ou má absorção intestinal; défices congénitos ou enzimáticos; convulsões major; hipotermia; asma severa; intoxicação por monóxido de carbono; feocromocitoma e doença maligna metastática.

Foram selecionados 843 doentes, dos quais 622 apresentaram critérios de exclusão. Foram incluídos 83 doentes não diabéticos e 138 doentes diabéticos, dos quais 65 encontravam-se medicados com metformina. O total de 221 doentes estudados incluiu 106 (47,8\%) homens e 115 $(52,2 \%)$ mulheres, com idades médias de $75 \pm 11,6$ anos (37-97 anos).

Foi recolhida informação referente ao motivo de ida à urgência (alterações inespecíficas do estado de consciência/ patologia do trato urinário/ patologia cardio-respiratória/ patologia gastrointestinal/ síndrome febril sem foco/ patologia dermatológica/ patologia ortopédica), antecedentes documentados de insuficiência cardíaca (IC), doença pulmonar obstrutiva crónica (DPOC) ou obesidade, tratamento farmacológico habitual (com especificação das respetivas doses), pressão arterial de entrada $(\mathrm{mmHg})$,-e destino dos doentes após observação (alta /internamento /consulta / óbito). A obesidade foi definida como índice de massa cor- 
poral $(\mathrm{IMC}) \geq 30 \mathrm{Kg} / \mathrm{m}^{2}$.

Foram reunidos os dados obtidos a partir das análises efetuadas em contexto assistencial: pressão arterial de oxigénio (paO2), pressão arterial de dióxido de carbono (paCO2), concentração de bicarbonato ( $\mathrm{HCO} 3)$, lactatos, glicemia, creatinina, azoto ureico, alanina transaminase $(A L T)$, aspartato transaminase (AST), gama glutamil transpeptidase (GGT) e proteína $C$ reativa ultrassensível (PCR). O valor normal da concentração plasmática de lactatos (dentro do intervalo de referência) foi definido como uma concentração entre 0,0 e 2,0 mmol/L. Hiperlactacidemia foi definida para valores de lactatos $\geq 2 \mathrm{mmol} / \mathrm{l}$, com hiperlactacidemia significativa se valores $\geq 4 \mathrm{mmol} / \mathrm{L}$. Acidose láctica foi definida como $\mathrm{pH}<7,35 \mathrm{com}$ lactatos $>4 \mathrm{mmol} / \mathrm{l}$ (com anion gap $\geq 15 \mathrm{mEq} / \mathrm{l}$ em combinação com acidose metabólica ou mista).

\section{Análise estatística}

Na caraterização global da amostra as variáveis numéricas foram apresentadas através da média \pm desvio padrão e as variáveis qualitativas recorrendo às frequências absolutas e relativas. Para as análises univariadas utilizou-se o teste de independência do Qui-Quadrado, e o teste $\mathrm{t}$ de Student para amostras independentes. Para análise dos objetivos do estudo utilizou-se a regressão linear, com o método Stepwise, para verificar quais as variáveis que têm um efeito estatisticamente significativo nos lactatos e utilizou-se a regressão logística, com o método Forward,

Tabela 1 - Comparação das caraterísticas clinicas entre doentes diabéticos e não diabéticos

\begin{tabular}{|c|c|c|c|c|c|}
\hline & \multicolumn{2}{|c|}{$\begin{array}{l}\text { Não Diabético } \\
\quad(N=83)\end{array}$} & \multicolumn{2}{|c|}{$\begin{array}{l}\text { Diabético } \\
(N=138)\end{array}$} & \multirow[t]{2}{*}{$p$} \\
\hline & $N$ & $\%$ & $\mathbf{N}$ & $\%$ & \\
\hline \multicolumn{6}{|l|}{ Sexo } \\
\hline Masculino & 40 & $48,2 \%$ & 66 & $47,8 \%$ & 0,958 \\
\hline Feminino & 43 & $51,8 \%$ & 72 & $52,2 \%$ & \\
\hline Idade (M $\pm S D)$ & \multicolumn{2}{|c|}{$74,7 \pm 13,7$} & \multicolumn{2}{|c|}{$75,3 \pm 10,2$} & 0,767 \\
\hline \multicolumn{6}{|l|}{ Motivo de ida à Urgência } \\
\hline Alterações inespecíficas do estado de consciência/bem-estar & 14 & $17,1 \%$ & 43 & $31,2 \%$ & $0,049^{*}$ \\
\hline Patologia do trato urinário & 7 & $8,5 \%$ & 10 & $7,2 \%$ & \\
\hline Patologia cardiorrespiratória & 56 & $68,3 \%$ & 67 & $48,6 \%$ & \\
\hline Patologia gastrointestinal & 4 & $4,9 \%$ & 10 & $7,2 \%$ & \\
\hline Síndrome febril sem foco & 1 & $1,2 \%$ & 4 & $2,9 \%$ & \\
\hline Patologia dermatológica/ortopédica & 0 & $0,0 \%$ & 4 & $2,9 \%$ & \\
\hline \multicolumn{6}{|l|}{ Insuficiência Cardíaca } \\
\hline Não & 65 & $78,3 \%$ & 105 & $76,6 \%$ & 0,774 \\
\hline Sim & 18 & $21,7 \%$ & 32 & $23,4 \%$ & \\
\hline \multicolumn{6}{|l|}{ DPOC } \\
\hline Não & 65 & $78,3 \%$ & 116 & $85,3 \%$ & 0,226 \\
\hline Sim & 18 & $21,7 \%$ & 20 & $14,7 \%$ & \\
\hline \multicolumn{6}{|l|}{ Obesidade } \\
\hline Não & 31 & $73,8 \%$ & 46 & $55,4 \%$ & $0,046^{*}$ \\
\hline $\operatorname{Sim}$ & 11 & $26,2 \%$ & 37 & $44,6 \%$ & \\
\hline TAS (M $\pm \mathrm{DP})$ & \multicolumn{2}{|c|}{$136 \pm 2,7$} & \multicolumn{2}{|c|}{$139,6 \pm 2,2$} & 0,309 \\
\hline TAD (M $\pm \mathrm{DP})$ & \multicolumn{2}{|c|}{$78,2 \pm 1,4$} & \multicolumn{2}{|c|}{$78,7 \pm 1,4$} & 0,842 \\
\hline \multicolumn{6}{|l|}{ Destino à data de alta } \\
\hline Alta & 60 & $72,3 \%$ & 79 & $57,2 \%$ & 0,066 \\
\hline Internamento & 22 & $26,5 \%$ & 58 & $42,0 \%$ & \\
\hline Falecido & 1 & $1,2 \%$ & 1 & $0,7 \%$ & \\
\hline
\end{tabular}

${ }^{\star} p<0,05 ;{ }^{*} p<0,01$; M: Média; DP: Desvio Padrão 
para determinar quais os fatores que influenciam a hiperlactacidemia e o destino. A curva ROC (receiving operating caracteristic) foi utilizada para determinar o ponto de corte ideal de creatinina na identificação de hiperlactacidemia significativa. A análise estatística foi realizada com o software IBM SPSS versão 21.0 (SPSS; IBM Corporation, New York, New York, USA). $P<0,05$ foi considerado estatisticamente significativo.

\section{RESULTADOS}

\section{Caraterísticas da população do estudo}

As Tabelas 1 e 2 apresentam os resultados referentes à análise univariada das diferentes variáveis em estudo (demográficas, clínicas, analíticas e tratamento) considerando o doente ser ou não diabético. Em relação ao motivo de deslocação à urgência $68,3 \%$ dos não diabéticos apresentaram queixas cardiorrespiratórias, enquanto nos diabéticos essa proporção foi de 48,6\%; as alterações inespecíficas do estado de consciência ocorreram em 17,1\% dos doentes não diabéticos e em $31,2 \%$ dos doentes diabéticos. Observou-se uma maior percentagem de obesidade nos diabéticos (44,6\% vs. $26,2 \%)$. Os doentes diabéticos apresentaram níveis médios de creatinina $(1,4 \pm 0,1$ vs. $1,1 \pm 0,1, p<0,05)$ e de glicemia $(184,1 \pm 7,8$. vs. $114,0 \pm 2,4$, $p<0,01)$ superiores. Os níveis médios de lactacidémia foram significativamente superiores nos doentes diabéticos $(2,1 \pm 0,1$ vs. $1,1 \pm 0,1)$ bem como a proporção de casos de hiperlactacidemia $(39,1 \%$ vs. $3,6 \%)$.

$\mathrm{Na}$ Tabela 3 apresenta-se a caraterização dos indivíduos diabéticos segundo o tratamento ou não com metformina, enfocando as diferenças identificadas entre o grupo de estudo e grupo de controlo. Um elevado número de doentes diabéticos não fazia medicação antidiabética e só 66 doentes se encontravam medicados com metfomina. Os doentes medicados com metformina apresentaram-se significativamente mais novos $(73,5 \pm 1,3$ vs. 76,9 $\pm 1,2)$, com níveis médios de creatinina inferiores (1,2 $\pm 0,1$ vs. $1,5 \pm 0,1)$, e níveis médios de ácido láctico significativamente superiores $(2,7 \pm 0,2$ vs. $1,6 \pm 0,1)$. Nos doentes que não faziam metformina apenas $23,3 \%$ apresentaram hiperlactacidemia, comparativamente a $56,9 \%$ daqueles que faziam metformina $(p<0,001)$.

A distribuição do lactato plasmático nos dois grupos encontra-se representada na Fig. 1.

Foram contabilizados 5 casos de acidose láctica, todos em doentes tratados com metformina, e em contexto de falência renal aguda. A Tabela 4 representa o tratamento antidiabético instituído nos doentes em estudo. Os doentes sob metformina faziam, em média, $1568 \pm 533,5$ (500 3000) mg por dia.

\section{Fatores que influenciam os níveis de lactatos}

Na análise por regressão logística - variável dependente: lactacidémia verificou-se que os doentes diabéticos sob metformina apresentaram uma probabilidade de hiperlactacidemia 25 vezes superior $(\mathrm{OR}=25,1 ; p<0,01)$ face aos demais doentes, com a obesidade (OR $=9,15 ; p<0,05) \mathrm{e}$ o valor de glicemia $(O R=1,02 ; p<0,05)$ a aumentar essa probabilidade, e, quanto maior a TA $(\mathrm{OR}=0,93$; $p<0,05)$ menor a probabilidade de hiperlactacidemia.

Analisando especificamente os doentes diabéticos sob metformina, a correlação de Spearman revelou que os níveis de ácido láctico se encontraram positivamente correlacionados com a dose de metformina $(r=0,49, p<0,01)$,

Tabela 2 - Comparação das caraterísticas bioquímicas entre doentes diabéticos e não diabéticos

\begin{tabular}{|c|c|c|c|c|c|}
\hline & \multicolumn{2}{|c|}{$\begin{array}{l}\text { Não Diabético } \\
\quad(N=83)\end{array}$} & \multicolumn{2}{|c|}{$\begin{array}{l}\text { Diabético } \\
(N=138)\end{array}$} & \multirow{2}{*}{$p$} \\
\hline & $N$ & $\%$ & $N$ & $\%$ & \\
\hline Lactatos (M $\pm \mathrm{SD})(\mathrm{mmol} / \mathrm{L})$ & \multicolumn{2}{|c|}{$1,1 \pm 0,1$} & \multicolumn{2}{|c|}{$2,1 \pm 0,1$} & $0,001^{* *}$ \\
\hline \multicolumn{6}{|l|}{ Hiperlactacidemia } \\
\hline Não & 80 & $96,4 \%$ & 84 & $60,9 \%$ & $0,001^{\star *}$ \\
\hline Sim & 3 & $3,6 \%$ & 54 & $39,1 \%$ & \\
\hline Creatinina (M $\pm \mathrm{DP})(\mathrm{mg} / \mathrm{dL})$ & \multicolumn{2}{|c|}{$1,1 \pm 0,7$} & & $\pm 1,1$ & 0,013 \\
\hline Azoto Ureico (M \pm DP) (mg/dL) & \multicolumn{2}{|c|}{$27,9 \pm 2,4$} & & $\pm 2,1$ & 0,113 \\
\hline Glicemia (M \pm DP) (mg/dL) & \multicolumn{2}{|c|}{$114 \pm 2,4$} & & $\pm 7,8$ & 0,001 \\
\hline AST (M \pm DP) (U/L) & \multicolumn{2}{|c|}{$27,4 \pm 1,8$} & & $\pm 1,6$ & 0,587 \\
\hline ALT (M \pm DP) (U/L) & \multicolumn{2}{|c|}{$24,4 \pm 2,5$} & \multicolumn{2}{|c|}{$22,3 \pm 1,6$} & 0,456 \\
\hline GGT (M $\pm \mathrm{DP})(\mathrm{U} / \mathrm{L})$ & \multicolumn{2}{|c|}{$58,4 \pm 8,4$} & \multicolumn{2}{|c|}{$43,7 \pm 3,6$} & 0,110 \\
\hline$P C R(M \pm D P)(m g / d L)$ & \multicolumn{2}{|c|}{$3,3 \pm 0,5$} & & $\pm 0,6$ & 0,135 \\
\hline
\end{tabular}

${ }^{*} p<0,05 ;{ }^{*} p<0,01$; M: Média; DP: Desvio Padrão 
Tabela 3 - Comparação das caraterísticas clinicas e bioquímicas entre doentes diabéticos com e sem metformina

\begin{tabular}{|c|c|c|c|c|c|}
\hline & \multicolumn{2}{|c|}{$\begin{array}{l}\text { Sem tratamento } \\
\text { com Metformina } \\
\qquad(N=73)\end{array}$} & \multicolumn{2}{|c|}{$\begin{array}{l}\text { Com tratamento } \\
\text { com Metformina } \\
\qquad(N=65)\end{array}$} & \multirow[t]{2}{*}{$p$} \\
\hline & $N$ & $\%$ & $N$ & $\%$ & \\
\hline \multicolumn{6}{|l|}{ Sexo } \\
\hline Masculino & 33 & $45,2 \%$ & 33 & $50,8 \%$ & 0,514 \\
\hline Feminino & 40 & $54,8 \%$ & 32 & $49,2 \%$ & \\
\hline Idade (M \pm SD) & \multicolumn{2}{|c|}{$76,9 \pm 10,1$} & \multicolumn{2}{|c|}{$73,5 \pm 10,1$} & $0,046^{\star}$ \\
\hline \multicolumn{6}{|l|}{ Obesidade } \\
\hline Não & 24 & $58,5 \%$ & 22 & $52,4 \%$ & 0,573 \\
\hline Sim & 17 & $41,5 \%$ & 20 & $47,6 \%$ & \\
\hline Lactatos $(\mathrm{M} \pm \mathrm{SD})(\mathrm{mmol} / \mathrm{L})$ & \multicolumn{2}{|c|}{$1,6 \pm 0,1$} & \multicolumn{2}{|c|}{$2,7 \pm 0,2$} & $0,001^{* *}$ \\
\hline \multicolumn{6}{|l|}{ Hiperlactacidemia } \\
\hline Não & 56 & $76,7 \%$ & 28 & $43,1 \%$ & $0,001^{* *}$ \\
\hline Sim & 17 & $23,3 \%$ & 37 & $56,9 \%$ & \\
\hline Creatinina (M $\pm \mathrm{DP})(\mathrm{mg} / \mathrm{dL})$ & \multicolumn{2}{|c|}{$1,5 \pm 0,9$} & \multicolumn{2}{|c|}{$1,2 \pm 1,1$} & 0,048 \\
\hline Glicemia (M \pm DP) (mg/dL) & \multicolumn{2}{|c|}{$181,5 \pm 10,2$} & \multicolumn{2}{|c|}{$187 \pm 12$} & 0,724 \\
\hline
\end{tabular}

* $p<0,05 ;{ }^{*} p<0,01 ;$ M: Média; DP: Desvio Padrão

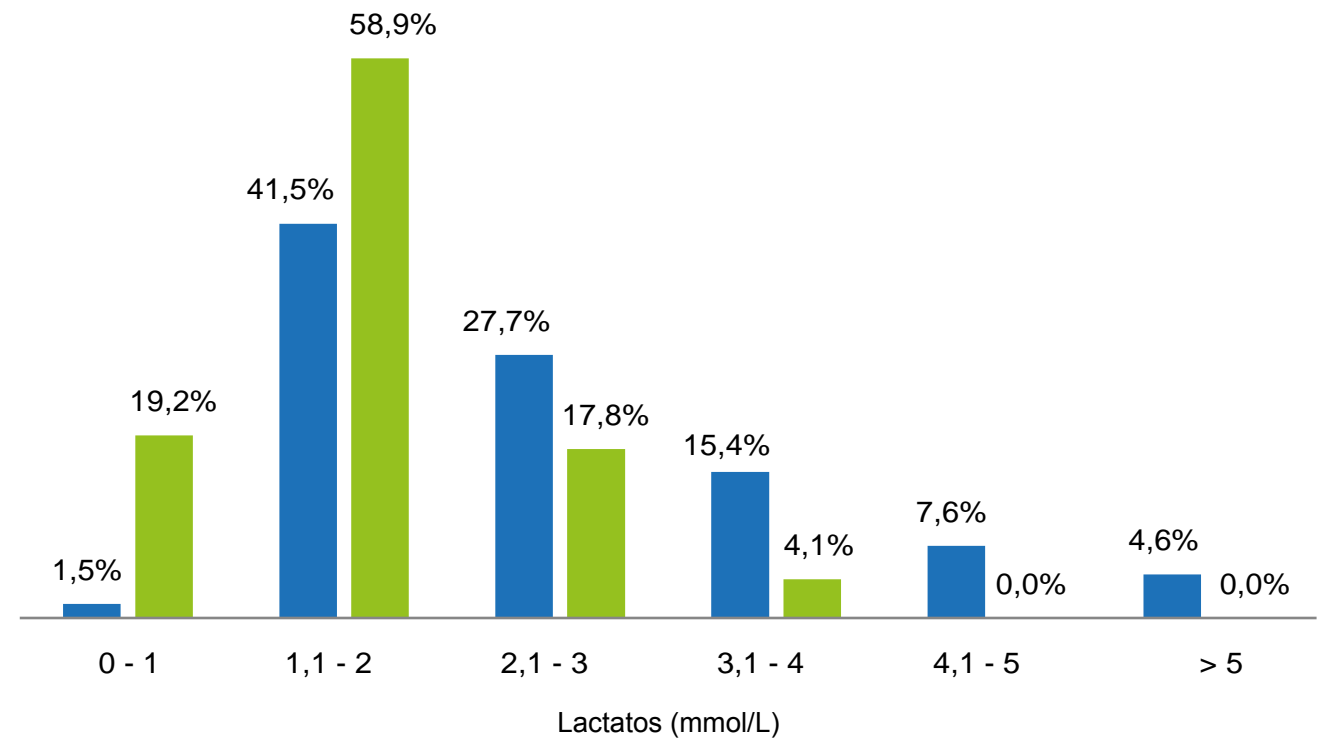

Figura 1 - Histograma de frequências mostrando a distribuição por percentagem de acordo com o valor de lactatos em doentes não tratados com metformina (verde) e tratados com metformina (azul)

os níveis de creatinina $(r=0,267, p<0,05)$ e de glicemia $(r=0,23, p<0,01)$; e negativamente com o valor de $\mathrm{pH}$ $(r=-0,381, p<0,01)$ e com a concentração arterial de HCO3 $(r=-0,558, p<0,01)$. Ausência de correlação significativa com a idade, fármacos instituídos, a pressão arterial de 02 e CO2, AST, ALT, GGT, PCR, TAS e TAD. No entanto, a análise de regressão múltipla stepwise mostrou que a creatinina representava o único fator independente a influenciar os valores de lactatos $(B=1,33 ; p<0,05)$ nestes doentes. 
Tabela 4 - Tratamento antidiabético dos doentes diabéticos observados no SU

\begin{tabular}{|c|c|c|}
\hline Tratamento da Diabetes & $N$ & $\%$ \\
\hline Não farmacológico & 99 & 44,8 \\
\hline Metformina & 30 & 13,6 \\
\hline Sulfonilureia & 11 & 5,0 \\
\hline Inibidor da DPP4 & 3 & 1,4 \\
\hline Insulina & 41 & 18,6 \\
\hline Metformina + Inibidor da DPP4 & 16 & 7,2 \\
\hline Metformina + Inibidor da DPP4 + Insulina & 3 & 1,4 \\
\hline Metformina + Insulina & 4 & 1,8 \\
\hline Metformina + Sulfonilureia + Acarbose & 3 & 1,4 \\
\hline Metformina + Sulfonilureia & 6 & 2,7 \\
\hline Metformina + Inibidor da DPP4 + Sulfonilureia & 4 & 1,8 \\
\hline Insulina + Inibidor da DPP4 & 1 & 0,5 \\
\hline
\end{tabular}

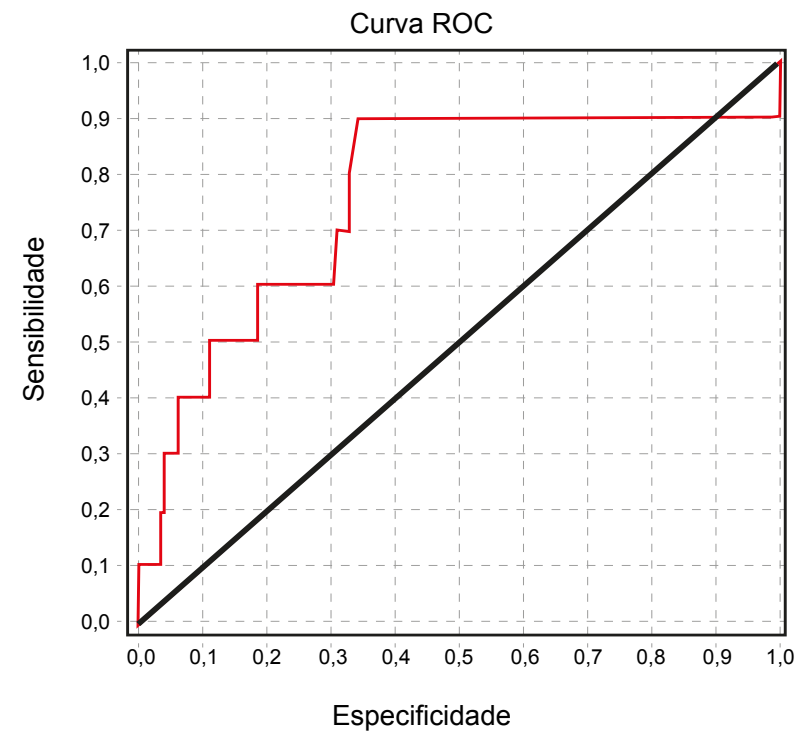

Figura 2 - Curva ROC (Receiver Operating Characteristic) dos valores de creatinina para detetar hiperlactacidemia significativa em doentes diabéticos tratados com metformina

\section{Efeito dos níveis de creatinina nos valores de lactatos}

Foi elaborada uma curva ROC, com AUC (area under curve) para determinação do ponto de corte de creatinina para a determinação da hiperlactacidemia significativa tendo sido utilizados os métodos de otimização, índice de Younden e o ponto na curva ROC mais próximo a $(0,1)$, para a determinação do referido valor. A AUC estimada foi $0,766$ (Cl 95\%: 0,584 - 0,936; $p=0,005)$ e o ponto de cor- te definido foi a creatinina de 1,145 mg/dl, tendo-se obtido uma sensibilidade de $90,0 \%$ e especificidade de $66 \%$, para prever a hiperlactacidemia significativa (Fig. 2).

\section{Efeito da hiperlactacidemia no prognóstico dos doen- tes}

Por último analisam-se quais os fatores que influenciam de forma estatisticamente significativa o destino dos doentes (internamento ou óbito vs. alta). A maioria dos doentes teve alta para o domicílio $(51,4 \%, n=113)$, com pouco mais de um terço $(35 \%, n=77)$ destes a necessitar de internamento e praticamente todos os restantes doentes a serem referenciados para seguimento em consulta externa $(12,7 \%, n=28)$. Apenas se registaram dois óbitos $(0,9 \%$, $n=2)$.

Verificou-se que nestes doentes a presença de infeção, traduzida pela elevação dos valores de PCR (OR = 1,10; IC95\%: 1,01 - 1,19: $p<0,01$ ) e a hiperlactacidemia (OR $=4,37$; IC95\%: 1,71 - 11,82: $p<0,05)$ influenciaram negativamente, e de forma independente, o prognóstico dos doentes, condicionando maior probabilidade do doente ficar internado ou falecer. No que refere á hiperlactacidemia, a sua presença aumentou em 4,4 vezes a probabilidade de internamento ou falecimento em relação aos doentes que apresentavam valores de lactatos dentro dos limites da normalidade. Mais ainda, os doentes com hiperlactacidemia que foram internados, apresentaram maior tempo de internamento $(21,66 \pm 5,86$ dias vs 13,68 $\pm 5,33$ dias, $p<0,001)$ e maior número de óbitos durante o internamento $(12,5 \%(n=4)$ vs $4,3 \%(n=2), p<0,05)$. 


\section{DISCUSSÃO}

Estudos prévios demonstraram um aumento em $45 \%$ no índice basal de turnover do lactato nos doentes diabéticos, o que se correlacionaria com um aumento da sua concentração plasmática. ${ }^{14}$ De fato, na nossa amostra, os doentes diabéticos apresentaram valores de lactatos significativamente superiores aos restantes doentes observados no SU. Acresce o fato de que, apesar de serem significativamente mais novos e apresentarem valores de creatinina sérica inferiores, os diabéticos medicados com metformina apresentaram níveis de lactatos significativamente superiores aos dos que se encontravam sob outros regimes terapêuticos, concluindo-se assim, que o efeito da metformina no metabolismo da glicose e do lactato aumenta significativamente a propensão para desenvolver hiperlactacidemia nos doentes diabéticos.

Além da terapêutica antidiabética instituída, os valores plasmáticos de lactatos foram correlacionados com múltiplas outras variáveis. A associação entre hiperlactacidemia e obesidade já tinha sido descrita noutros estudos. ${ }^{9,15}$ O mecanismo não se encontra totalmente elucidado, mas parece resultar de um ambiente tecidular deficiente em oxigénio, induzido pela obesidade e pela insulinorresistência, que, através da ativação da glicólise anaeróbia, condiciona um aumento da produção e libertação de ácido láctico pelo tecido adiposo. ${ }^{9}$ Por outro lado, verificou-se ainda uma correlação positiva entre os níveis séricos de lactatos e de glicose. Esta associação poderá ter por explicação uma diminuição da perfusão renal decorrente do aumento da diurese osmótica induzida pela hiperglicemia. ${ }^{16}$

Desta forma, o aumento do valor plasmático de lactatos nos doentes com DMT2 poderá resultar da conjugação entre uma diminuição global da capacidade oxidativa secundária a alteração do metabolismo dos lactatos, na qual o tratamento com metformina desempenharia um papel determinante, com um aumento da libertação de ácido láctico pelo tecido adiposo e diminuição da sua excreção renal.

Analisando especificamente os doentes diabéticos sob metformina, verificamos que, apesar de mais de metade dos doentes apresentarem valores de lactatos superiores a $2 \mathrm{mmol} / \mathrm{L}$, apenas foram contabilizados cinco casos que cumpriam os critérios de acidose láctica, o que limita a utilidade clinica da lactacidémia como indicador do risco de acidose nestes doentes. No presente estudo, todos os casos de acidose láctica associada à metformina ocorreram em indivíduos com intercorrências agudas, que, por si só, poderiam causar acidose. Porém, o valor de bicarbonato sérico ao correlacionar-se de forma negativa com o valor de lactatos, à semelhança do que acontece com o valor de $\mathrm{pH}$, poderá ser utilizado como marcador de acidose tecidular, e como um indicador simples do estado ácido-base. Justificando que apesar do valor de lactato plasmático ser insuficiente para monitorizar o risco de acidose láctica, a avaliação do equilíbrio ácido base deve ser efetuado para cada caso, de forma a minimizar o risco de desenvolvimento de acidose. Continuam a ser publicados relatos de casos de acidose láctica associada á metformina, que se reves- tem de grande importância, dada a elevada mortalidade associada; nalguns estudos, atinge os $50 \% .{ }^{17}$

$\mathrm{Na}$ nossa amostra, a lactacidémia apresentou ainda uma correlação positiva com a dose diária de metformina, pressupondo um maior risco de hiperlactacidemia em diabéticos sob doses terapêuticas superiores. No entanto, quando efetuado o ajuste para todas as variáveis referidas, e à semelhança do verificado em outros estudos, ${ }^{18}$ seria a utilização do fármaco independentemente da dose utilizada a antecipar o risco de hiperlactacidemia. Reforçando que, em contexto de intercorrências agudas ou hiperlactacidemia, o fármaco deve ser suspenso, uma vez que a redução da sua dose provavelmente será insuficiente.

A taxa de filtração glomerular estimada não deve ser aplicada em contexto de urgência, ${ }^{19} \mathrm{e}$, desta forma, o valor de creatinina mantém-se como marcador mais fiável e reprodutível da função renal. Na nossa análise a creatinina representou o único fator independente a influenciar o valor de lactatos nos doentes diabéticos medicados com metformina, com um aumento gradual da lactacidémia de acordo com os níveis de creatinina apresentados. O ponto de corte ideal para indicar hiperlactacidemia significativa foi de $1,145 \mathrm{mg} / \mathrm{dl}$, com sensibilidade de $90 \%$ e especificidade de $66 \%$, sugerindo que possa haver uma acumulação de lactatos antes do desenvolvimento de uma disfunção renal marcada. Tendo em conta que estes doentes foram observados no SU, não se pode excluir a existência de alteração prévia da função renal não conhecida ou documentada. De fato, alguns estudos demostraram que a acumulação de metformina inicia-se de forma progressiva em situações em que contraindicações, como a insuficiência renal, não foram respeitadas. ${ }^{20}$ Uma acumulação crónica do fármaco associada a um evento precipitante agudo poderia justificar a maior propensão a hiperlactacidemia nestes doentes, mesmo na ausência de elevação franca dos níveis de creatinina.

A hiperlactacidemia traduz um desequilíbrio entre a produção e a utilização de lactato. Em conjunto com a presença de marcadores biológicos de infeção ou inflamação, documentados pelo valor de PCR, antecipou de forma independente a necessidade de tratamento em regime de internamento ou o falecimento dos doentes admitidos no SU. Este achado surgiu em concordância com outros estudos, que mostram que níveis elevados de lactatos condicionam um aumento da mortalidade e que a transição de lactacidémia fisiológica para patológica ocorre mesmo com valores inferiores a $2 \mathrm{mmol} / \mathrm{l} .{ }^{12}$ Nos doentes que foram internados, o valor de lactatos manteve-se como um importante marcador de risco. Tanto a concentração de lactatos como a sua tendência evolutiva parecem condicionar o prognóstico dos doentes em estado crítico. ${ }^{13} \mathrm{Na}$ nossa amostra verificou-se que os doentes com hiperlactacidemia apresentavam maior tempo de internamento e mais número de óbitos durante o internamento. Apesar do número de doentes que cumpria estas condições ser reduzido e de provavelmente se tratar de doentes com um maior número de comorbilidades associadas, os doentes com hiperlactacidemia na 
altura da admissão pareceram apresentar um risco acrescido para eventos adversos, justificando a necessidade de procurar e tratar outras causas reversíveis eventualmente coexistentes nestes doentes.

Como limitações ao nosso estudo apontamos o fato de os doentes serem observados durante um curto espaço de tempo e por diversos observadores. Ao efetuar a revisão dos processos clínicos foi verificado que a maioria correspondia a doentes sem seguimento prévio na nossa instituição, não estando disponível informação clínica relevante como: duração da doença, terapêutica antidiabética prévia, exatidão no IMC, função renal de base, entre outros. Tratando-se de indivíduos maioritariamente com idades compreendidas entre os 60 e os 80 anos, poderá haver limitação na generalização destes resultados à maioria da população diabética, apesar de no contexto social atual, uma grande percentagem dos doentes diabéticos tipo 2 se incluir na faixa etária da amostra. Futuramente poderá ser útil avaliar em ambulatório os doentes que apresentaram hiperlactacidemia, e verificar se os níveis de lactatos e as condicionantes dos mesmos se mantêm.

\section{CONCLUSÃO}

Apesar de um grande número de doentes se encontrar não medicado, em contexto de urgência os doentes diabéticos apresentaram um risco de hiperlactacidemia superior à população geral. Este risco mostrou ser particularmente acrescido quando analisados especificamente os doentes diabéticos sob metformina. A monitorização do doente diabético, especialmente da sua função renal, deve ser mais rigorosa em doentes medicados com metformina, particu- larmente quando obesos ou com mau controlo glicémico. Quando em contexto de urgência, é necessário avaliar de forma individualizada os níveis de lactatos e o equilíbrio ácido base. A creatinina sérica representou o único fator preditor independente dos níveis de lactatos. A presença de hiperlactacidemia antecipou de forma independente a necessidade de tratamento em regime de internamento ou o falecimento dos doentes diabéticos observados no SU, justificando a sua utilização como marcador de pior prognóstico.

\section{AGRADECIMENTOS}

Os autores agradecem a Isabel Fonseca, na sua função de Diretora do Serviço de Urgência dos Hospitais da Universidade de Coimbra - Centro Hospitalar e Universitário de Coimbra, pela sua disponibilidade e colaboração científica, sem a qual o presente trabalho não seria possível.

\section{CONFLITOS DE INTERESSE}

Os autores declaram não existir qualquer conflito de interesses relativamente ao presente artigo. Os autores declaram ainda que parte do presente estudo foi previamente apresentado em no XIV Congresso Português de Endocrinologia, 24 a 27 de janeiro de 2013, Porto e no 15th European Congress of Endocrinology, 27 April - 1 May 2013 Copenhagen.

\section{FONTES DE FINANCIAMENTO}

Não existiram fontes externas de financiamento para a realização deste artigo.

\section{REFERÊNCIAS}

1. Qaseem A, Humphrey LL, Sweet DE, Starkey M, Shekelle P. Oral pharmacologic treatment of type 2 diabetes mellitus: a clinical practice guideline from the American College of Physicians. Ann Intern Med. 2012;156:218-31.

2. Inzucchi SE, Bergenstal RM, Buse JB, Diamant M, Ferrannini E, Nauck $M$, et al. Management of hyperglycaemia in type 2 diabetes: a patientcentered approach. Position statement of the American Diabetes Association (ADA) and the European Association for the Study of Diabetes (EASD). Diabetologia. 2012;55:1577-6.

3. Standards of medical care in diabetes--2012. Diabetes Care. 2012;35:S11-63.

4. Rodbard HW, Jellinger PS, Davidson JA, Einhorn D, Garber AJ, Grunberger $\mathrm{G}$, et al. Statement by an American Association of Clinical Endocrinologists/American College of Endocrinology consensus panel on type 2 diabetes mellitus: an algorithm for glycemic control. Endocr Pract. 2009;15:540-59.

5. Viollet B, Guigas B, Sanz Garcia N, Leclerc J, Foretz M, Andreelli F. Cellular and molecular mechanisms of metformin: an overview. Clin Sci. 2012;122:253-70.

6. Kreisberg RA. Lactate homeostasis and lactic acidosis. Ann Intern Med. 1980;92:227-37.

7. Lalau JD. Lactic acidosis induced by metformin: incidence, management and prevention. Drug Saf. 2010;33:727-40.

8. Perrone J, Phillips C, Gaieski D. Occult metformin toxicity in three patients with profound lactic acidosis. J Emerg Med. 2011;40:271-5.

9. Liu F, Lu JX, Tang JL, Li L, Lu HJ, Hou XH, et al. Relationship of plasma creatinine and lactic acid in type 2 diabetic patients without renal dysfunction. Chin Med J. 2009;122:2547-53.

10. Franzetti I, Paolo D, Marco G, Emanuela M, Elisabetta Z, Renato U. Possible synergistic effect of metformin and enalapril on the development of hyperkaliemic lactic acidosis. Diabetes Res Clin Pract. 1997;38:173-6.

11. Chan NN, Fauvel NJ, Feher MD. Non-steroidal anti-inflammatory drugs and metformin: a cause for concern? Lancet. 1998;352:201.

12. Nichol AD, Egi M, Pettila V, Bellomo R, French C, Hart G, et al. Relative hyperlactatemia and hospital mortality in critically ill patients: a retrospective multi-centre study. Crit Care. 2010;14:R25.

13. Kruse O, Grunnet N, Barfod C. Blood lactate as a predictor for in-hospital mortality in patients admitted acutely to hospital: a systematic review. Scand J Trauma Resusc Emerg Med. 2011;19:74.

14. Cusi K, Consoli A, DeFronzo RA. Metabolic effects of metformin on glucose and lactate metabolism in noninsulin-dependent diabetes mellitus. $\mathrm{J}$ Clin Endocrinol Metab.1996;81:4059-67.

15. Crawford SO, Ambrose MS, Hoogeveen RC, Brancati FL, Ballantyne $\mathrm{CM}$, Young $\mathrm{JH}$. Association of lactate with blood pressure before and after rapid weight loss. Am J Hypertens. 2008;21:1337-42.

16. Pepper GM, Schwartz M. Lactic acidosis associated with Glucophage use in a man with normal renal and hepatic function. Diabetes Care. 1997;20:232-3

17. Bruijstens LA, van Luin M, Buscher-Jungerhans PM, Bosch FH. Reality of severe metformin-induced lactic acidosis in the absence of chronic renal impairment. Neth J Med. 2008;66:185-90.

18. Davis TM, Jackson D, Davis WA, Bruce DG, Chubb P. The relationship between metformin therapy and the fasting plasma lactate in type 2 diabetes: The Fremantle Diabetes Study. Br J Clin Pharmacol. 2001;52:137-44

19. Martin JH, Fay MF, Ungerer JP. eGFR--use beyond the evidence. Med J Aust. 2009;190:197-9.

20. Ekström N, Schiöler L, Svensson AM, Eeg-Olofsson K, Miao Jonasson $\mathrm{J}$, Zethelius B, et al. Effectiveness and safety of metformin in 51675 patients with type 2 diabetes and different levels of renal function: a cohort study from the Swedish National Diabetes Register. BMJ Open. 2012;2: pii: e001076. 


\section{Relação entre o Tratamento com Metformina e o Desenvolvimento de Hiperlactacidemia no Serviço de Urgência Acta Med Port 2014:27:196-203}

Publicado pela Acta Médica Portuguesa, a Revista Científica da Ordem dos Médicos

Av. Almirante Gago Coutinho, 151

1749-084 Lisboa, Portugal.

Tel: +351218428215

E-mail: submissao@actamedicaportuguesa.com

www.actamedicaportuguesa.com

ISSN:0870-399X | e-ISSN: 1646-0758

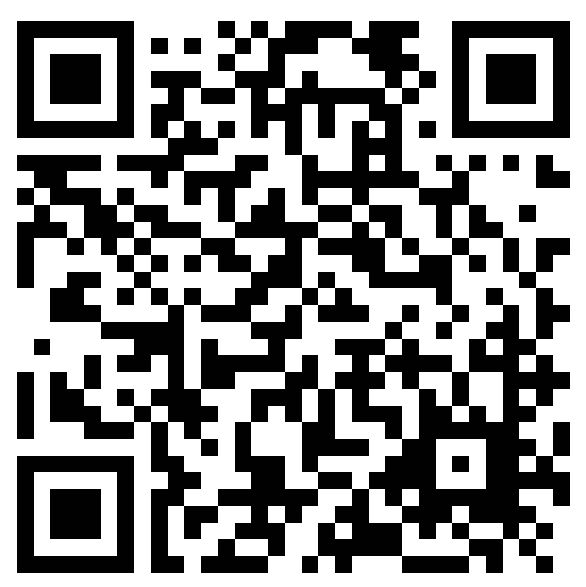

